\title{
The role of perceptual organization in the depth perception of kinetic lattice displays
}

\author{
WILLIAM BALCH and ROBERT SHAW \\ University of Connecticut, Storrs, Connecticut 06268
}

\begin{abstract}
College student subjects were asked to judge perceived depth in computer-generated displays. In all displays, one lattice of points moved through a stationary lattice in either a rowwise or columnwise direction. No points of the two lattices ever touched. Two display variables, strain and shear, each had a significant effect on depth ratings. Shear, however, was only effective at the level of strain for which depth ratings were high. The results confirm earlier studies in which "topological breakage" information was found to affect depth perception. The outcome of this study suggests that principles of perceptual organization can influence the nature of effective breakage information.
\end{abstract}

The study of information used in the perception of depth has often been guided by a cue-oriented approach (e.g., Künnapas, 1968; Shiffman, 1976). Such an orientation can be characterized generally as follows: There are a number of cues, each analyzed separately and each contributing additively to the perception of depth (although the various contributions may be weighted differently). These cues, with the exception of motion parallax, are static in that they are defined in terms of nonmoving visual displays.

However, some depth effects using kinetic displays (e.g., Börgesson \& Von Hofsten, 1972; Kaplan, 1969; Wallach \& O'Connell, 1953) are difficult to account for in terms of standard depth cues. Of particular relevance to the present study are Mace's (1971) displays (further reported by Mace \& Shaw, 1974).

These cases all consisted of two rectangular lattices of points generated by a computer on a CRT screen. At least one lattice moved across the screen. It will suffice here to consider only those cases where one lattice moved and the other remained stationary. To assess what conditions yielded pronounced depth perception, Mace and Shaw manipulated several geometric variables to form a series of displays.

This study was completed while the first author was a graduate student and the second author a faculty member at the University of Minnesota. Preparation of this paper was supported in part by grants to the University of Minnesota, Center for Research in Human Learning, from the National Science Foundation (GB$35703 \mathrm{X}$ ), the National Institute of Child Health and Human Development (HD-01136 and HD-0098), and the Graduate School of the University of Minnesota. The authors gratefully acknowledge the assistance of Edward Rapoport and Michael Huck and the helpful comments of William Mace, Leonard Mark, and James Todd. Correspondence concerning the article or requests for reprints should be sent to William Balch, Department of Psychology, University of Connecticut, Storrs, Connecticut 06268.
Subjects' spontaneous descriptions revealed that the displays varied in how often they were described in terms of depth. Though the exact nature of the depth reports varied, a common type of report indicated that each lattice was seen as a co-planar pattern, and that one pattern, which could be either lattice, was perceived in front of the other. The depth effect obtained was thus indeterminantly ordered.

A variable which Mace and Shaw called "phase" was found to have an effect on subjects' numerical ratings of the clarity of depth seen in a given display. In Figure 1A, the moving lattice, which has points with attached arrows to indicate direction of motion, is "in phase" with the stationary one. Note that the points of the former will periodically become superimposed on those of the latter. On the other hand,
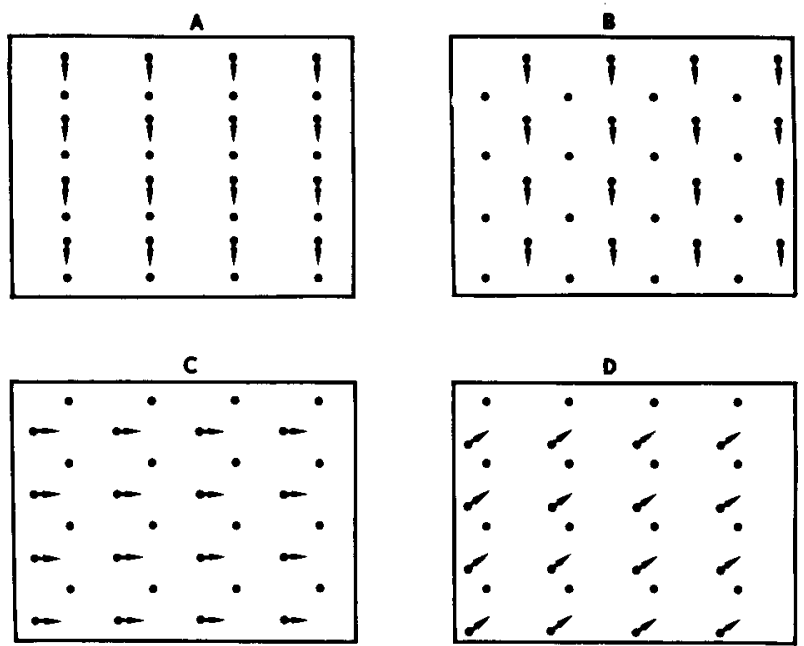

Figure 1. Illustrative lattice displays (Mace \& Shaw, 1974). (A) In phase, vertical; (B) out of phase, vertical; (C) out of phase, horizontal; (D) out of phase, diagonal. 
Figure 1B shows a case where the moving lattice is "out of phase" with the stationary one. Note that the columns of the former move between the columns of the latter, with no contact at all among the points of the two lattices. It was found that "out-of-phase" displays were rated higher on depth than "in-phase" displays (Mace, 1971; Mace \& Shaw, 1974).

Direction of motion also had an effect on depth ratings. Figures $1 \mathrm{~B}, 1 \mathrm{C}$, and $1 \mathrm{D}$ are all out-of-phase displays, but the direction taken by the moving lattice varies: vertical (B), horizontal (C), or diagonal (D). Diagonal motion was a more effective depth condition than horizontal motion, which was, in turn, more effective than vertical.

Neither the direction of motion nor the phase effect can be adequately explained in terms of brightness, texture, motion parallax, or accretion/deletion variables, since these variables were held approximately constant across all displays. Thus an important question remains as to what kind of information best accounts for the effects.

A type of kinetic information called topological breakage, originally applied to the perception of indeterminantly ordered depth separation between the shadows of two randomly textured surfaces (Gibson, Gibson, Smith, \& Flock, 1959), appears to explain some of these effects. By breakage information, Gibson et al. (1959) meant the change in adjacent order of texture elements, taken together with the nonchange in the order within the subset of elements belonging to each of the two surfaces. One more specific formulation of breakage information has been proposed by Mace (1971), and applied by him to the phase variable of his lattice displays. This formulation was based on the number of times a point of one lattice disrupted (i.e., passed between, in any direction) a two-point line contained in a cell of the other lattice. Each disruption counts as one breakage. Figure 2 illustrates, for the various displays

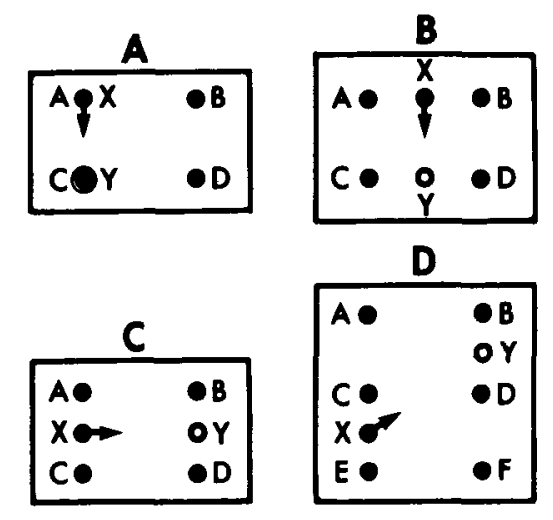

Figure 2. Analysis of displays shown in Figure 1. A single point of the moving lattice is considered as it moves from position $X$ to position $Y$ (open circle). Points of the stationary lattice are labeled to show the various two-point lines broken by the moving point. depicted in Figure 1, the lines disrupted by a single moving lattice point, $X$, as it moves through a cell of the stationary lattice to position $Y$, indicated by an open circle. For in-phase lattices, illustrated in Figure 2A, only one line is broken-AC, in this case. When the display shown in Figure $2 \mathrm{~A}$ is made out of phase (see Figure 2B), four lines are disrupted: $A B$, $A D, B C$, and $C D$. Likewise, four lines $(A C, A D, B C$, and $B D$ ) are disrupted in the horizontal out-of-phase display, shown in Figure 2C. Thus, the effect of phase on depth ratings can be accounted for in terms of the relative number of breakages in out-of-phase vs. in-phase displays.

Mace's (1971) analysis can be extended to account for the high depth ratings given to diagonal motion displays. Figure 2D illustrates the motion of a diagonal lattice point as it passes through the stationary lattice. Two cells of the latter lattice are necessary in this case, since the intention of the analysis is to consider one period of motion. (That is, the diagonally moving point shown in Figure 2D starts at position $X$, and does not reach a comparable place in the stationary lattice structure until position Y.) It can be seen from Figure 2D that five breakages are involved in the diagonal motion case $(C E, C F, C D, A D$, and $\mathrm{BD})$, as opposed to four in the vertical and horizontal cases illustrated in Figures $2 \mathrm{~B}$ and $2 \mathrm{C}$.

An important problem with this simple version of topological breakage is that it does not allow for the influence of Gestalt and similar contextual principles, according to which the perception of an entire display is organized. An example of this problem is the fact that greater depth was seen, by Mace's (1971) and Mace and Shaw's (1974) subjects, in the horizontal displays than in the vertical ones. According to the context-free analysis discussed, there are no topological breakage differences between the vertical and horizontal cases (see Figures 2B and 2C).

Although horizontal motion may have been inherently more effective than vertical motion in inducing depth perception in these displays, another explanation for the effect is proposed here. This explanation hinges on the point that the lattices used by Mace (1971) and by Mace and Shaw (1974) were rectangular, being about $37 \%$ longer along the horizontal dimension than along the vertical one. This stretching along one axis will be called here a "strain" of the lattices. The display shown in Figure 1B represents a strain that is perpendicular to the vertical direction of motion in this figure, because the strain is horizontal. Due to the operation of the Gestalt law of proximity, this display should be seen as a set of columns moving parallel to-or "sliding through" -a stationary set of columns. Since the alternate columns appear to be moving alongside adjacent stationary columns, the columnwise organization of the display is not disrupted. The display shown in Figure $1 \mathrm{C}$ has the same horizontal strain, but the mo- 

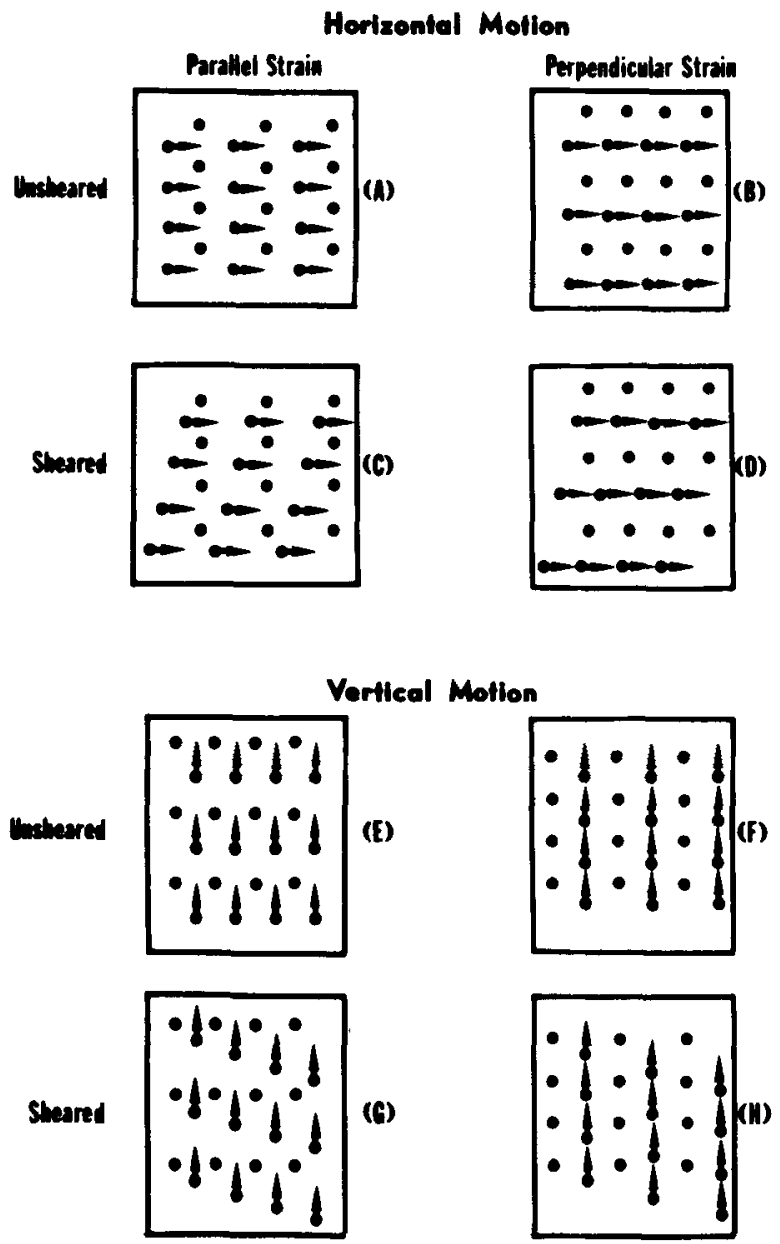

Figure 3. The eight experimental displays.

tion is now horizontal too. The direction of motion and the strain are now parallel to one another. In this case, the columnwise organization of each lattice is disrupted as the moving columns appear to break through stationary ones. If the disruption of the columns constitutes the relevant breakage in these strained displays, then one would expect greater depth in Figure $1 C$, where the direction of strain is parallel to that of motion.

The variables of strain and direction of motion covaried in Mace's displays. To determine whether direction of motion per se was responsible for the finding that the horizontal motion yielded clearer depth perception than did the vertical, Mace ran an additional experiment using square lattices, so that no strain was present in the displays. The superiority of the horizontal direction was no longer significant under these conditions (although it did approach significance). In the present experiment, strain and direction of motion were varied independently. While there is no strong empirical or theoretical basis for predicting a direction of motion effect, strain should have an effect on depth ratings because of its Gestalt influence on breakage information.
Another Gestalt-like influence on topological breakage is illustrated in Figure 3. This figure shows the displays used in the present study. Comparing Figure $3 \mathrm{~A}$ and $3 \mathrm{C}$, it can be seen that in the latter the horizontally moving columns are positionally tilted"sheared" in the present terminology-with respect to the stationary ones. When analyzed in the manner illustrated in Figure 2, the sheared and unsheared displays are equivalent in amount of topological breakage. However, in the unsheared display shown in Figure 3A, entire columns of the moving lattice cut simultaneously through stationary columns, creating only periodic breakage. In the display pictured in Figure 3C, the breakage is more continuous, since different points move through the stationary columns at different times. In this sense, the sheared display involves more breakage and should induce clearer perception of depth.

The independent variables in this experiment are direction of motion, strain, and shear. The focus of this study is on how strain and shear affect depth ratings of the lattice displays due to the operation of Gestalt influences on topological breakage. The two hypotheses can be summarized as follows: (a) Straining the lattices of a display along the axis parallel to the direction of the moving lattice, as in Figures $3 \mathrm{~A}$ and $3 \mathrm{E}$, should yield relatively high depth ratings. (b) When such a strain is applied, additional clarity of depth can be induced by shearing the moving lattice, as in Figures $3 \mathrm{C}$ and $3 \mathrm{G}$.

\section{METHOD}

\section{Subjects}

Six female and four male undergraduates enrolled in the introductory psychology course at the University of Minnesota participated in this experiment. They received a small amount of academic credit for being subjects.

\section{Apparatus}

All displays were generated by a PDP-12 computer and presented on an auxiliary cathode ray tube (CR7), Display Type UR-12, located in a separate room. The room was dimiy illuminated, with sufficient light for the experimenter to record each subject's depth ratings. A plywood box, open at one end and having a built-in viewing window at the other, was clamped to the CRT. The window contained two light-polarizing filters, adjusted to reduce the intensity of the points to a constant and comfortable viewing level. The subject's visual field was restricted to the screen by a short tunnel-like headrest, through which the subject looked with both eyes. The viewing distance to the screen was about $41 \mathrm{~cm}$. The screen itself was covered by a piece of black cardboard with a circular hole having a diameter of about $14.5^{\circ}$ in visual angle. This mask hid the corners of the approximately square displays generated by the computer, so that the central $75 \%$ of the display was visible. It should be pointed out that pilot subjects, viewing the displays before the circular mask was introduced, reported being distracted by "blinking" at the edges of the displays (i.e., the simultaneous appearance or disappearance of rows or columns of moving lattice points). The mask effectively avoided this distraction. 


\section{Displays and Experimental Design}

All displays consisted of one lattice moving through another stationary lattice. The lattices consisted of luminous (green) points generated on a dark screen. For each lattice, the columns were spaced $1.8^{\circ}$ apart and the rows, $.9^{\circ}$ apart, or vice versa, with the longer spacing being between the rows. The size of each lattice was $8 \times 16$ points, the larger number of points occurring along the dimension having the shorter spacing.

Three display variables, each having two levels, were factorially combined for a total of eight displays. In describing the variables, the illustrations of Figure 3 will be referred to. These illustrations show the essential geometric properties of the displays. However, it should be remembered that the displays actually seen by the subjects had a circular boundary, and that the lattices had considerably more points than those shown in Figure 3.

The variables and their levels were as follows: (a) Direction of motion: The direction of the moving lattice points was either horizontal (left-to-right motion, illustrated in Figures 3A-3D) or vertical (bottom-to-top motion, shown in Figures 3E-3H). Moving points were generated as necessary about $.5^{\circ}$ to the left or bottom of the stationary lattice. With the mask on the screen, moving and stationary points filled the circular visible display area at all times. ${ }^{1}$ (b) Strain: Half of the displays (see Figures 3A, 3C, 3E, and $3 \mathrm{G}$ ) had a parallel strain. The column or row spacing of both lattices were twice as great along the axis parallel to the direction of the moving points. In these displays, it appeared that moving columns or rows were "breaking" through stationary columns or rows. For the other displays (see Figures 3B, 3D, $3 \mathrm{~F}$, and $3 \mathrm{H}$ ), the strain was perpendicular. Here the column or row spacings of the lattices were twice as large along the axis perpendicular to the direction of moving lattice points. The appearance in these displays was that moving rows (or columns) were "sliding" through stationary rows (or columns) without any breakage. (c) Shear: In sheared displays (see Figures 3C, 3D, $3 \mathrm{G}$, and $3 \mathrm{H}$ ), moving columns (or rows) were tilted $14^{\circ}$ clockwise. Note that as a result there is more or less continuous breakage in the two sheared displays with parallel strain (see Figures $3 \mathrm{C}$ and $3 \mathrm{G}$ ). That is, different points of the moving columns (or rows) break through stationary columns (or rows) at different times. On the other hand, in unsheared displays, the columns and rows of both lattices are untilted, remaining vertical or horizontal (see Figures 3A, 3B, 3E, and 3F).

\section{Procedure}

Subjects were run individually. Each subject viewed the entire series of displays three times, in a different random order each time. The experimenter did not know which particular display was being viewed at any given time.

Before the present experiment was undertaken, 6 out of 10 pilot subjects had spontaneously described at least some of the displays in the series in terms of depth. (Three out of the remaining four reported having noticed depth in some displays after being asked if they had seen any depth during their viewing of series. Additionally, pilot subjects were shown the same displays with no motion present, and none spontaneously described these static versions in terms of depth). Since almost all the pilot subjects had no difficulty viewing the series of kinetic displays in terms of clarity of depth, subjects in the present study were asked to view the displays for strength of the depth effect.

The subjects were initially told that they would be viewing a series of displays consisting of dots. They were then instructed to study each display and observe whether the array of dots appeared to lie on the same plane or whether some of the dots appeared to lie in front of or behind other dots. The subjects were told that in cases where a depth separation was observed, they would be asked to rate the strength of the depth effect experienced. Additional instructions were given to encourage them to look at the whole display, and to avoid concentrating on any particular subset of dots. Viewing time for each display was approximately
$7 \mathrm{sec}$. After each display, there was a period of about $3 \mathrm{sec}$ in which the screen was dark while the next display was being computed.

After viewing the series once, the subjects were asked to rate each display on the clarity of depth separation. A 0-to-6 numerical scale was used, with 0 being defined as a flat display and 6 as a display having the maximum of clear separation. The instructions emphasized that it did not matter which points appeared in front or behind. Additionally, the subjects were asked not to concentrate on the exact distance perceived between points. Rather, they were told to make their judgments qualitatively, based only on the clarity of the depth impression in each display. It was also pointed out that the scale had seven numbers, with " 3 " being exactly the intermediate value. The subjects were advised that ratings above or below 3 should indicate relatively strong or weak depth, respectively. They were instructed that 0 alone was to be used to indicate a judgment of no depth at all. Finally, they were told to use the full range of the scale if possible, giving the least separated-looking display a relatively low rating, and the most separated looking display a relatively high rating. The subjects rated each display for two successive series. As soon as a subject gave a rating on a particular display (which usually took 1 to $3 \mathrm{sec}$ ), the experimenter recorded the rating and continued to the next display of the randomly ordered series.

\section{RESULTS}

The ratings of the last series were used in the main statistical analysis. However, the consistency of subjects from the first to the second series of ratings was also checked by computing the correlation between a subject's rating of a display during the first series and the same subject's rating of that display during the second series-across all eight displays. This correlation was significant $(\mathrm{r}=+.80, \mathrm{p}<.001)$.

Table 1 gives the means and standard deviations of subjects' ratings of the eight displays comprising the final series. A three-way repeated-treatment analysis of variance was applied to the ratings.

The largest main effect was that of strain $[F(1,9)$ $=40.54, \mathrm{p}<.001]$. Displays with a strain parallel to the direction of motion were rated relatively high in clarity of depth, for an average of 4.0 on the scale. However, displays with a perpendicular strain were

Table 1

Means and Standard Deviations of the Depth Ratings for the Experimental Displays

\begin{tabular}{lllll}
\hline & & \multicolumn{2}{c}{ Strain } \\
\cline { 3 - 5 } $\begin{array}{l}\text { Direction } \\
\text { of Motion }\end{array}$ & & & \multicolumn{2}{c}{$\begin{array}{c}\text { Perpen- } \\
\text { dicular }\end{array}$} \\
\hline \multirow{4}{*}{ Horizontal } & Unsheared & Mean & 3.7 & 1.3 \\
& & SD & 1.16 & .83 \\
& Sheared & Mean & 4.9 & 1.4 \\
& & SD & 1.10 & .97 \\
& Unsheared & Mean & 2.9 & 1.7 \\
& & SD & 1.52 & 1.06 \\
& & Mean & 4.4 & 1.5 \\
& Sheared & SD & 1.90 & 1.08 \\
\hline
\end{tabular}

Note-Minimum rating $=0$. Maximum rating $=6$. Means and standard deviations are each based on 10 scores. 
rated low, averaging 1.5 . The most effective strain condition thus occurred when moving columns (or rows) appeared to break through stationary ones.

The other main effect found was that of shear $[F(1,9)=7.76, p<.05]$. The mean depth rating of sheared displays was 3.1 , while ratings of unsheared displays averaged only 2.4 . However, the real source of this effect was the significant interaction between strain and shear $[F(1,9)=13.67, p<.01]$.

For displays with parallel strain, sheared displays had a higher mean rating (4.7) than unsheared ones (3.3). On the other hand, there was no effect of shear for displays with perpendicular strain. Sheared and unsheared displays alike averaged a low 1.5 rating.

Direction of motion was not significant as a main effect $[F(1,9)<1, p>.10]$ and did not interact significantly with the other independent variables.

\section{DISCUSSION}

The results support both of the hypotheses proposed: (1) Displays where strain is parallel to the direction of the moving lattice produce higher depth ratings than do displays where strain is perpendicular. This effect was expected because, for parallel strain displays, moving lines appear to break through stationary lines, due to Gestalt proximity. (2) Parallel strain displays in which the lines doing the breaking are sheared yield higher depth ratings than do parallel strain displays where there is no shear. This effect was expected because tilting these lines makes the breakage relatively continuous rather than periodic. The principle involved here seems to be analogous to what Gestaltists call "good continuity." Though this term has generally been applied to static figures, it might also be applied to patterns defined over both space and time. In parallel strain patterns that are unsheared (Figures $3 \mathrm{~A}$ and $3 \mathrm{E}$ ), the breakage not only was periodic, but appeared abrupt when it occurred. Pilot subjects sometimes reported that the moving lines appeared to slow down considerably as they broke through stationary ones, as if the latter were "resisting"' the motion of the former. No sharp discontinuities were reported for sheared cases.

The outcome of this experiment supports the conclusion of Gibson et al. (1959) that breakage information can be an important determinant of depth perception. The results suggest that the nature of this information depends on organizational principles affecting the perception of an entire display. The analysis discussed earlier, involving the breaking of two point lines by a third point, is a simple and relatively context-free formulation that can explain some of the effects found in kinetic lattice research (Mace, 1971; Mace \& Shaw, 1974). In this experiment, the number of breakages, according to this formulation, was held constant. The variables manipulated here affected the way each entire display was perceptually organized. The obtained effects of these variables on depth perception suggest that principles of organization can determine the nature of operative breakage information in a display. This information, in turn, affects depth perception.

The effects found here cannot be adequately explained in terms of other types of depth information. In particular, motion parallax is an information source that may have contributed in general to the depth seen in these displays. However, the effects studied here concern differences between displays. Motion parallax (one lattice moving while the other does not) was constant across all displays. Thus breakage information, as prescribed by principles of perceptual organization, can be reasonably assumed to account for differences in clarity of depth among these displays.

An interesting aspect of the depth seen in these displays was its indeterminate ordering. (This assertion is based on informal observation and questioning of subjects during pilot research, but the same observation has been made in other studies involving topological breakage: Gibson et al., 1959; Mace, 1971.) Why does this information apparently specify separation in depth, but not its order? Hopefully, a solution to this problem can be found through further experimental and theoretical analysis of topological breakage, especially in relation to other formulations (accretion/deletion and motion parallax, for instance) which are generally considered as kinds of information capable of providing an ordered depth effect.

It should also be pointed out that the mere presence of breakage is not in itself sufficient for a clear impression of even indeterminate depth. "In-phase" displays (Mace, 1971; Mace \& Shaw, 1974), for instance, represent a case where some breakage is present but depth ratings are low (see Figure 1A). The units of breakage and the continuity and number of breakages are all important in determining clarity of depth perception. This study has shown that such considerations depend on principles of perceptual organization.

Much is known about what kinds of information are sufficient for depth perception. However, it is not yet known whether or not there are necessary conditions for perceiving depth, or what they might be if they exist. To answer these questions, it is important to study the interrelations between already known types of information for depth.

\section{REFERENCES}

Börgesson, E., \& Von Hofsten, C. Spatial determinants of depth perception in two-dot motion patterns. Perception \& Psychophysics, 1972, 11, 263-268.

Gibson. E. J.. Gibson, J. J.. Smith, O. W., \& Flock, H. R. 
Motion parallax as a determinant of perceived depth. Journal of Experimental Psychology, 1959, 58, 40-51.

KaPLAN, G. Kinetic disruption of optical texture: The perception of depth at an edge. Perception \& Psychophysics, 1969, 6. 193-198.

Künnapas, T. Distance perception as a function of available visual cues. Journal of Experimental Psychology, 1968, 77, 523-529.

MACE, W. An investigation of spatial and kinetic information for separation in depth using computer generated dot patterns. (Doctoral dissertation, University of Minnesota, 1971). Dissertation Abstracts International, 1972, 32, 6687-B. (University Microfilms No. 72-14, 333)

MACE, W. H., \& SHAW, R. Simple kinetic information for transparent depth. Perception \& Psychophysics, 1974, 15. 201-209.

Schiffman, H. Sensation and perception: An integrated approach. New York: Wiley, 1976.
Wallach, H., \& O'Connell, D. The kinetic depth effect. Journal of Experimental Psychology, 1953, 45, 205-217.

\section{NOTE}

1. The moving lattice had a velocity of $1.8^{\circ} / \mathrm{sec}$, and moved for the time required for the observer to make a judgment on a given display. The motion of this lattice was accomplished by changing its position 20 times per second. To the experimenters, the motion looked continuous; and none of the pilot or experimental subjects who commented on the displays said anything to indicate that this motion looked discrete or "apparent."

(Received for publication October 24, 1977; revision accepted March 28, 1978.)

\section{ERRATUM}

Hellströ, Å. Factors producing and factors not producing time errors: An experiment with loudness comparisons. Perception \& Psychophysics, 1978, 23, 433-444-The last sentence on page 434 should correctly read as follows:

Denoting by $Z_{1}$ and $Z_{2}$ the standard normal deviates which correspond to the probability $P_{1}$ for a judgment to fall in category " 1, " and the probability $P_{2}$ for it to fall in category "2" [i.e., $Z_{1}=$ $\Phi^{-1}\left(P_{1}\right)$ and $\left.Z_{2}=-\Phi^{-1}\left(P_{2}\right)\right]$, we may note that

$$
Z_{1}=(D-T-b) / o_{c}
$$

and that

$$
Z_{2}=(D+T-b) / o_{c}
$$

